\title{
THE (DOUBLE) WHITE DWARF BINARY SDSS 1257+5428
}

\author{
S. R. KulKarni AND M. H. van KerkwiJK ${ }^{1}$ \\ Caltech Optical Observatories 249-17, California Institute of Technology, Pasadena, CA 91125, USA \\ Received 2010 March 10; accepted 2010 June 9; published 2010 July 27
}

\begin{abstract}
SDSS $1257+5428$ is a white dwarf in a close orbit with a companion that has been suggested to be a neutron star. If so, it hosts the closest known neutron star, and its existence implies a great abundance of similar systems and a rate of white dwarf neutron-star mergers similar to that of the type Ia supernova rate. Here, we present high signal-to-noise spectra of SDSS $1257+5428$, which confirm an independent finding that the system is in fact composed of two white dwarfs, one relatively cool and with low mass and the other hotter and more massive. With this, the demographics and merger rate are no longer puzzling (various factors combine to lower the latter by more than 2 orders of magnitude). We show that the spectra are fit well with a combination of two hydrogen model atmospheres, as long as the lines of the higher-gravity component are broadened significantly relative to what is expected from just pressure broadening. Interpreting this additional broadening as due to rotation, the inferred spin period is short, about 1 minute. Similarly rapid rotation is only seen in accreting white dwarfs that are magnetic; empirically, it appears that in non-magnetized white dwarfs, accreted angular momentum is lost by nova explosions before it can be transferred to the white dwarf. This suggests that the massive white dwarf in SDSS $1257+5428$ is magnetic as well, with $B \simeq 10^{5} \mathrm{G}$. Alternatively, the broadening seen in the spectral lines could be due to a stronger magnetic field, of $\sim 10^{6} \mathrm{G}$. The two models can be distinguished by further observations.
\end{abstract}

Key words: binaries: close - stars: individual (SDSS J125733.63+542850.5) - white dwarfs

Online-only material: color figures

\section{SDSS $1257+5428$}

An unexpected return from the Sloan Digital Sky Survey (SDSS; York et al. 2000) has been the discovery of white dwarf binaries with short periods by Badenes et al. (2009) and Mullally et al. (2009). These authors took advantage of the fact that the multiplexed SDSS spectroscopic observations consist of several 15 minute integrations, which can be analyzed individually to look for rapid changes in radial velocity.

The first finds are already quite interesting. SDSS $\mathrm{J} 143633.29+501026.8$ (orbital period, $P_{\mathrm{b}}=1.15 \mathrm{hr}$ ) and SDSS J105353.89+520031.0 $\left(P_{\mathrm{b}}=0.96 \mathrm{hr}\right)$ are both low mass $\left(\sim 0.3 M_{\odot}\right)$ DA white dwarfs orbiting an unseen companion. For both systems, the measured mass function can be reasonably explained by invoking a white dwarf for the secondary. ${ }^{2}$ The orbital periods are so short that the systems are expected to merge within a Hubble time. These two binaries increased the toll of such interesting double degenerates to seven (Mullally et al. 2009). The number is likely to increase further, e.g., from a targeted search of low-mass white dwarfs found in SDSS, Kilic et al. (2010) find four binaries (including the two from Mullally et al. 2009). These authors also discuss in detail the fate of these systems: since the combined masses are likely below the Chandrasekhar mass, most will not explode as type Ia supernovae, but rather, depending on whether the mass transfer is stable or not, become very close cataclysmic variables (CVs) similar to AM CVn systems or hydrogen-deficient giant stars similar to R CrB stars.

SDSS J125733.63+542850.5 (SDSS 1257+5428 hereafter) consists of a DA white dwarf primary in an orbit with pe-

\footnotetext{
1 On sabbatical leave from Department of Astronomy and Astrophysics, University of Toronto, 50 St. George Street, Toronto, ON M5S 3H4, Canada.

2 We refer to the detected DA white dwarf as the photometric primary (or "primary") and the unseen/fainter companion as the photometric secondary ("secondary").
}

riod $P_{\mathrm{b}}=4.6 \mathrm{hr}$ and no measurable eccentricity. The inferred companion mass (as constrained by the spectroscopically inferred white dwarf mass and the mass function) is above the Chandrasekhar mass (in a probabilistic sense). If so, the companion is either a neutron star or a black hole, and, at the estimated distance, $d \approx 50 \mathrm{pc}$, it would be the nearest ultra-compact object known; see Badenes et al. (2009).

The importance of the proximity of SDSS $1257+5428$ becomes apparent when we consider the distances to the nearest members of different sub-classes of neutron stars (see Table 1): the nearest millisecond binary pulsar (a binary consisting of a low-mass white dwarf and a pulsar, and in a circular orbit), PSR J0437-4715; the thermally X-ray emitting middle-aged neutron star, RX J1856.5-3754; the nearest long period pulsar, PSR J2144-3933; the nearest ordinary pulsar(s), PSR J0108-1431 and PSR 0950+08; the nearest young pulsar, Vela; and the nearest $\gamma$-ray pulsar, Geminga. (For the ordinary pulsars, accounting for the usual beaming factor of 0.1 results in a distance of about $130 \mathrm{pc}$.) The distance $d_{\mathrm{NS}} \sim 150 \mathrm{pc}$ for the nearest neutron star(s) is in accord with the global demographics of neutron stars as well as demographics of specific classes of neutron stars (e.g., Lyne et al. 1998; Kaplan \& van Kerkwijk 2009 and references therein).

Specifically for SDSS $1257+5428$, if its unseen companion is truly a neutron star then, given the short period, the system would arguably be a binary millisecond pulsar. For millisecond pulsars, the local density is between 30 and $45 \mathrm{kpc}^{-3}$ (Cordes \& Chernoff 1997). The expected distance to the nearest millisecond pulsar is thus 175-200 pc, consistent with the observations (i.e., with PSR J0437-3715), but inconsistent with the proximity of SDSS $1257+5428$.

Thus, a neutron-star companion to SDSS $1257+5428$ would be very puzzling, unless one assumes that its proximity to the solar system is a statistical fluke. Motivated by this as well as by the alarming implications of a paper by Thompson et al. (2009), 
Table 1

Nearest Neutron Stars

\begin{tabular}{lcc}
\hline \hline \multicolumn{1}{c}{ Name } & $\begin{array}{c}\text { Distance } \\
(\mathrm{pc})\end{array}$ & Ref. \\
\hline PSRJ0437-4715 & $156.3 \pm 1.3$ & Deller et al. 2008 \\
RXJ1856.5-3754 & $161_{-14}^{+18}$ & van Kerkwijk \& Kaplan 2007 \\
PSRJ2144-3933 & $165_{-14}^{+17}$ & Deller et al. 2009 \\
PSRJ0108-1431 & $240_{-61}^{+124}$ & Deller et al. 2009 \\
PSR0950+08 & $260_{-5}^{+58}$ & Brisken et al. 2002 \\
Vela pulsar & $286_{-17}^{+19}$ & Dodson et al. 2003 \\
Geminga & $250_{-62}^{+120}$ & Faherty et al. 2007 \\
\hline
\end{tabular}

we first reconsider the case for a neutron-star companion. A reading of the Badenes et al. (2009) paper shows that it rests primarily on the high mass inferred for the primary, with kinematics offered as a supporting argument. We revisit these two issues in the next two sections and then present observations showing that the system in fact hosts two white dwarfs.

Before proceeding, we note that while writing up our results, a preprint by Marsh et al. (2010) appeared, which presented spectra in which the presence of a second component is also evident. These authors discuss in detail the preceding and future evolution of this binary. In this paper, we focus on the demographics and on obtaining more accurate constraints on the white dwarfs in the system.

\section{REVISITING THE MASS OF THE COMPANION}

Badenes et al. (2009) present radial-velocity variations of SDSS $1257+5428$. The velocity data appear to be of high quality and so we accept the two inferences: orbital period, $P_{\mathrm{b}}=4.555 \mathrm{hr}$ and a velocity semimajor amplitude of the primary, $K_{1}=322.7 \pm 6.3 \mathrm{~km} \mathrm{~s}^{-1}$. The uncertainty in $P$ is virtually negligible. The mass function is thus

$$
\mathcal{M}_{2}=\frac{M_{2}^{3} \sin ^{3} i}{\left(M_{1}+M_{2}\right)^{2}}=\frac{P_{\mathrm{b}} K_{1}^{3}}{2 \pi G}=0.66 \pm 0.04 M_{\odot},
$$

where the uncertainty of $5.9 \%$ of $\mathcal{M}_{2}$ arises from cubing $K_{1}$. The minimum mass of the unseen companion is $\mathcal{M}_{2}$ (obtained by assuming that the system is seen edge-on and setting $M_{1}=0$ ).

Badenes et al. (2009) infer a mass for the primary based on fitting the Balmer absorption lines to model atmospheres of DA white dwarfs and find $M_{1}=0.92_{-0.32}^{+0.28} M_{\odot}$ (the large uncertainty in the inferred mass is due to the poor model fit to the data; more detail on this issue is given below). As noted by Badenes et al. (2009), this implies that the unseen companion is quite massive (for a white dwarf); there is a $92 \%$ change that $M_{1}>0.64 M_{\odot}$ and that $M_{2}$ exceeds the Chandrasekhar limit.

One issue is that empirically it has been found that spectroscopic masses inferred for cool white dwarfs $\left(T_{\text {eff }}<12,000 \mathrm{~K}\right)$ are larger by about $10 \%$ than the true masses. This has been a long-standing issue in the field of white dwarfs, but with no resolution in sight; see Tremblay et al. (2010) and Koester et al. (2009a) for recent summaries. Spectral fitting yields $P=g / \kappa$, where $P$ is the gas pressure, $\kappa$ is the mass opacity, and $g$ is the local acceleration due to gravity. It has been suspected that in cool white dwarfs, which have convective atmospheres, helium might be dredged up. This would pollute the atmosphere and decrease $\kappa$ (relative to a pure hydrogen atmosphere). This hypothesis would then explain why the masses inferred spectroscopically are systematically higher for cool DA white dwarfs. Sensitive measurements in field white dwarfs, however, have failed to detect the expected He I features at $5876 \AA$ (Tremblay et al. 2010).

The above might suggest that the white dwarf mass has been overestimated, thus weakening the case for a neutron-star companion. On the other hand, recently Tremblay \& Bergeron (2009) showed that the prescription for the broadening of the hydrogen lines was insufficiently accurate, leading one to underestimate white dwarf masses (see also Falcon et al. 2010). Overall, it appears that there is a systematic uncertainty of about $10 \%$ in the masses derived from model-atmosphere fits.

More important than the uncertainties in the models is that the spectral fits to the data are poor. This is acknowledged by Badenes et al. (2009), but it is worth stressing that such poor fits are not routinely seen in DA white dwarfs (e.g., see Bergeron et al. 1992). That typical fits are good can also be seen from the small inferred uncertainties in $T_{\text {eff }}$ and $\log g$. Taking the SPY survey of high sensitivity and high spectral resolution observations of white dwarfs as an example and restricting ourselves to the subset of cool DA white dwarfs $(T<12,000 \mathrm{~K})$, we find that the largest uncertainty in $\log g$ is 0.03 (corresponding to $7 \%$ uncertainty) and the median 0.01 (corresponding to $2.3 \%$ ).

Thus, the poor fits for the Balmer lines of SDSS $1257+5428$ suggest that either SDSS $1257+5428$ has a strange pathology (in which case the modeling of the Balmer lines is fundamentally suspect) or the companion is contributing to the light. If the latter, it might be responsible for some of the wiggles seen in the continuum, or, if it is a DA white dwarf, contribute to the observed Balmer lines. From a more detailed look at the spectral fits of Badenes et al. (2009), one sees that these poorly match the narrow cores of lower Balmer lines and underpredict the higher Balmer lines. The presence of the higher Balmer lines is indicative of lower gravity, since these lines are very sensitive to pressure broadening (the size of a hydrogen atom is quadratically proportional to the upper level of the transition). Combined with the wide wings shown by the lower Balmer lines, which require high gravity, this supports the idea that the observed spectrum of SDSS $1257+5428$ is produced by more than one DA white dwarf.

\section{REVISITING THE KINEMATICS}

Pulsars, both single and binary, are noted for their large space motion (Cordes \& Chernoff 1997). The mean three-dimensional speed of the low-mass millisecond pulsars is $84 \mathrm{~km} \mathrm{~s}^{-1}$. Badenes et al. (2009) offer the kinematics of SDSS $1257+5428$ as a consistency argument for a neutron-star secondary.

The proper motion of SDSS $1257+5428$ is $51 \pm 5 \mathrm{mas} \mathrm{yr}^{-1}$ (Abazajian et al. 2009). At the stated distance of $50 \mathrm{pc}$, the proper motion translates to $12 \mathrm{~km} \mathrm{~s}^{-1}$, which is not particularly distinguishable. Badenes et al. (2009), from their velocities, find that the mean radial velocity of the primary is $\gamma_{1}=$ $-29 \pm 5 \mathrm{~km} \mathrm{~s}^{-1}$.

A compilation of the mean radial velocities ${ }^{3}$ of double degenerates listed by Nelemans et al. (2005) shows a spread of -36 to $70 \mathrm{~km} \mathrm{~s}^{-1}$. Correcting for the expected gravitational redshift ${ }^{4}$ for the primary, using the masses as obtained from

\footnotetext{
3 For consistency, we restricted the analysis to the radial velocity of the photometric primary.

4 Throughout this article, we use the mass-radius fitting formula of Nauenberg (1972) which provides a good approximation to the classical results of Hamada \& Salpeter (1961). The gravitational or Einstein redshift, converted to velocity, is $\gamma_{E}=0.633 M / R \mathrm{~km} \mathrm{~s}^{-1}$, where $M$ is the mass and $R$ is the radius.
} 
Table 2

Log of Observations and Radial-velocity Measurements

\begin{tabular}{ccccccc}
\hline \hline $\begin{array}{c}\text { Epoch } \\
(1)\end{array}$ & $\begin{array}{c}\text { Slit } \\
(2)\end{array}$ & $\begin{array}{c}\text { Camera } \\
(3)\end{array}$ & $\begin{array}{c}\tau \\
(4)\end{array}$ & $\begin{array}{c}\text { MJD } \\
(5)\end{array}$ & $\begin{array}{c}\phi \\
(6)\end{array}$ & $\begin{array}{c}v \\
(7)\end{array}$ \\
\hline $12: 13$ & 0.7 & $\mathrm{~B}$ & 780 & 0.51660 & 0.86 & \\
$12: 14$ & 0.7 & $\mathrm{R}$ & 340 & 0.51470 & 0.85 & $-292 \pm 3$ \\
$12: 21$ & 0.7 & $\mathrm{R}$ & 340 & 0.51945 & 0.87 & $-262 \pm 3$ \\
$12: 28$ & 1.0 & $\mathrm{~B}$ & 780 & 0.52727 & 0.92 & \\
$12: 28$ & 1.0 & $\mathrm{R}$ & 340 & 0.52491 & 0.90 & $-219 \pm 3$ \\
$12: 35$ & 1.0 & $\mathrm{R}$ & 340 & 0.52967 & 0.93 & $-173 \pm 3$ \\
$12: 42$ & 1.0 & $\mathrm{~B}$ & 780 & 0.53709 & 0.97 & \\
$12: 43$ & 1.0 & $\mathrm{R}$ & 340 & 0.53473 & 0.96 & $-119 \pm 3$ \\
$12: 49$ & 1.0 & $\mathrm{R}$ & 340 & 0.53950 & 0.98 & $-69 \pm 3$ \\
\hline
\end{tabular}

Notes. Column 1: exposure start time on 2010 February 11. Column 2: slit width in arcseconds. Column 3: camera (B: blue; R: red). Column 4: integration time in seconds. Column 5: barycentric mid-exposure time relative to MJD 55238.0. Column 6: orbital phase calculated using the ephemeris of Marsh et al. (2010), MJD 54845.67470(8) +0.18979154(9)E, where phase 0 corresponds to inferior conjunction of the photometric primary. Column 7: radial velocity calibrated relative to sky lines and corrected to the solar system barycenter. There may be systematic effects larger than the formal uncertainties due to inaccuracies in our correction for the high-gravity component. For the observations, we used the Atmospheric Dispersion Corrector (ADC) and set up LRIS as follows. A dichroic at 5600 Å separated the incoming light into a blue and a red channel. For the blue channel, we employed the 600 lines $\mathrm{mm}^{-1}$ grism, blazed at $4000 \AA$. The dispersion is $0.63 \AA$ per pixel and the spectral FWHM $4.1 \AA$ (for the 1 arcsecond slit). The detectors consisted of two $4096 \times 2048$ pixel Marconi CCDs with each $15 \mu \mathrm{m}$ square pixel covering $0{ }^{\prime} .135$ on the side. For the red channel, we used a grating with 1200 lines $\mathrm{mm}^{-1}$ and blazed at $7500 \AA$. The dispersion was $0.4 \AA$ per pixel and the spectral FWHM $2.1 \AA$ (for the 1 arcsecond slit). The detector for this channel was a mosaic of two LBNL CCDs, of the same size and with the same plate scale as the blue ones (but read out binned by two in the spatial direction)

spectroscopic modeling, one finds that the true radial velocities for these 24 double degenerates lie between -60 and $50 \mathrm{~km} \mathrm{~s}^{-1}$.

For the spectroscopic mass of $0.9 \pm 0.3 M_{\odot}$, the gravitational redshift (converted to velocity) is $60_{-30}^{+70} \mathrm{~km} \mathrm{~s}^{-1}$. The $1 \sigma$ lower bound results in $-59 \pm 5 \mathrm{~km} \mathrm{~s}^{-1}$ — within the expected kinematics of double degenerates.

\section{SPECTROSCOPIC OBSERVATIONS AND DATA REDUCTION}

On the night of UT 2010 February 11, we undertook observations of SDSS $1257+5428$ with the Low Resolution Imaging Spectrograph (LRIS) mounted on the Keck I 10 m telescope (Oke et al. 1995). The night was clear and the seeing typically about 0 '.7 (red camera) and 0.'8 (blue camera). The observations are summarized in Table 2. We started a sequence of observations of SDSS $1257+5428$ using a 0.7 slit and later changed to $1^{\prime \prime} 0$ to better match the seeing. Following this, we observed a number of internal light sources (incandescent for flat fields, $\mathrm{Hg} / \mathrm{Kr} / \mathrm{Zn} / \mathrm{Ar}$ and $\mathrm{Ne} / \mathrm{Ar}$ arc lamps for wavelength calibration), as well as, at a later time, observations of the flux standard Feige 34 (Oke 1990) with the same spectrograph setup.

For the reduction, we used the European Southern Observatory (ESO) Munich Image Data Analysis System (MIDAS) and routines running in the MIDAS environment. For all images, we subtracted bias as determined from the overscan regions. For the blue images, we subsequently corrected for small-scale variations in efficiency by dividing by a spatially averaged flat field, normalized using a third-degree polynomial, and with the bluest, poorly exposed part shortward of $4000 \AA$ A replaced by unity. For the red images, we divided by the flat field, normalized using a bi-linear fit. We extracted the spectra using an optimal extraction procedure similar to that of Horne (1986), after subtracting sky determined from neighboring regions.

Wavelength calibration was done using arc spectra. For the blue arm, fifth-degree polynomial fits were required to give adequate dispersion solutions, with typical rms residuals of $0.02 \AA$ (for 14 lines). For the red arm, a fifth-degree polynomial gave residuals of $\sim 0.017 \AA$ (for 27 lines). We used the same wavelength solution for all spectra, but corrected for possible drifts using the oxygen sky emission lines at 5577.340 and $6300.304 \AA$, in the blue and red, respectively. For the blue, the line is at the edge of our wavelength range, and hence our wavelength scale may be slightly off. Indeed, we find that the velocities inferred from the blue spectra show a slit-sizedependent offset from those inferred from the red spectra; hence, we will use the blue side only to (attempt to) measure relative velocities.

For flux calibration, we first corrected all spectra approximately for atmospheric extinction using a curve made by combining the Canada-France-Hawaii Telescope (CFHT) values (Bèland et al. 1988) shortward of $5200 \AA$ with the better sampled La Silla values long-ward of $5200 \AA$ A (ESO Users Manual 1993; see also Tug 1977). Next, for the blue spectra, we calculated response curves by comparing our observed spectra for Feige 34 with the calibrated spectra of Oke (1990) as provided by STScI: ${ }^{5}$ we slightly smoothed our spectra to match the resolution of the calibrated spectra, divided the two, and smoothly interpolated the ratio. Since all spectra were taken through a relatively narrow slit, this should give good relative calibration, but the absolute calibration may be off. Folding our flux calibrated spectra through the SDSS $u, g$, and $r$ response curves, ${ }^{6}$ we infer $g=16.6, u-g=0.41$, and $g-r=0.11$, which is in good agreement with the values listed by Badenes et al. (2009): $16.844 \pm 0.004,0.511 \pm 0.010$, and $0.112 \pm 0.007$, respectively, especially considering that our spectra do not completely cover the $u$ band. For plotting purposes, we rescaled our blue and red spectra to match the $g$ and $r$ magnitudes, respectively.

\section{SPECTRAL ANALYSIS}

The spectra show Balmer lines up to $\mathrm{H} 12$ and distinct asymmetries in some of the Balmer absorption features, with the red wing shallower than the blue one (Figure 1; note that there is no sign of wiggles in the continuum such as those seen in Figure 5 of Badenes et al. 2009). This suggests that two sources contribute to the Balmer features, with the second redshifted relative to the one responsible for the line cores. The asymmetry is most pronounced in $\mathrm{H} \delta$ and $\mathrm{H} \epsilon$ and less so for both lower and higher Balmer lines. The former suggests that the temperature of the second component is higher than that of the most prominent one (but that the emitting area is smaller), so that it contributes mostly at bluer wavelengths, while the latter suggests that its gravity must be higher, so that the higher Balmer lines are less strong.

Granted that there are two sources contributing to the Balmer series we were, nonetheless, puzzled by the absence of double line cores already noted by Badenes et al. (2009). After some experimentation and contemplation, we invoked rotational broadening for the secondary star.

\footnotetext{
5 ftp://ftp.stsci.edu/cdbs/calspec/

6 http://www.sdss.org/dr6/instruments/imager/index.html\#filters
} 


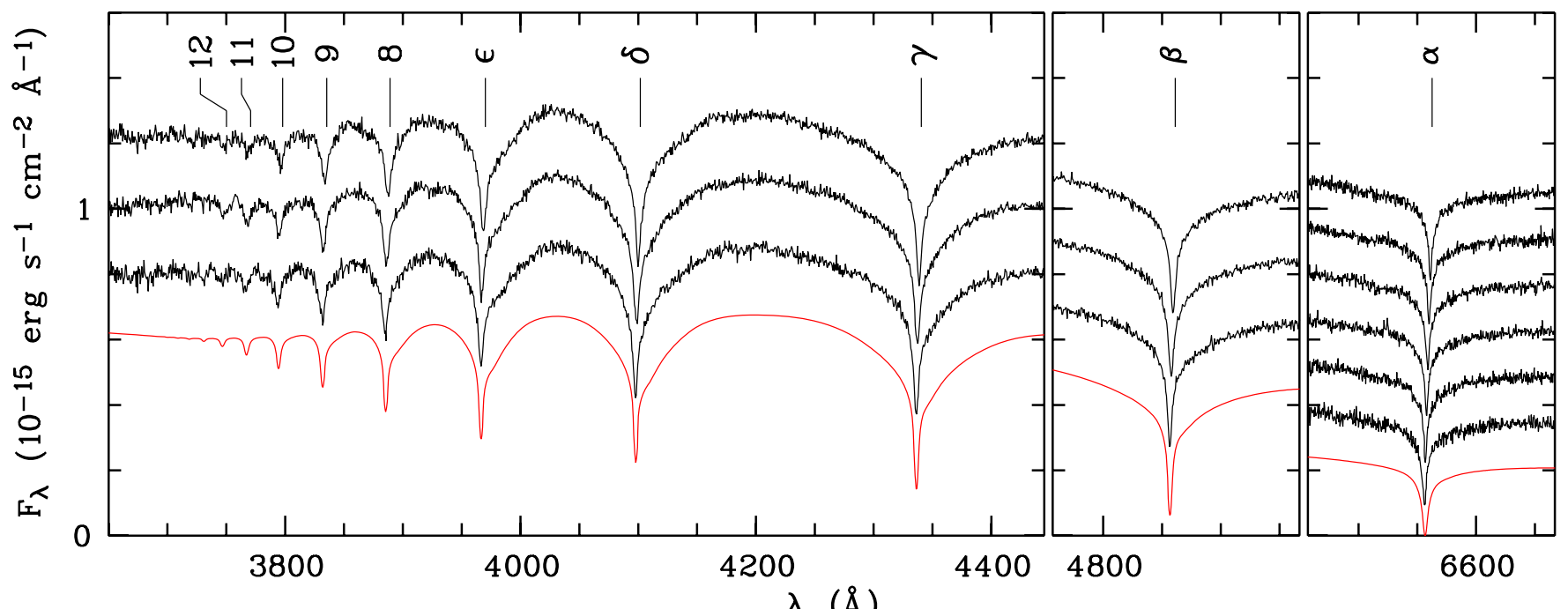

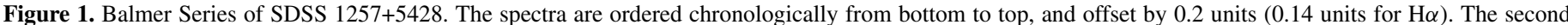

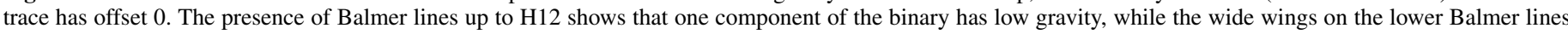

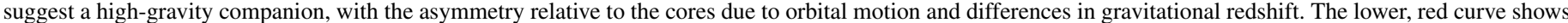
the model fit described in the text (the fit is to the first set of spectra; see also Figure 2).

(A color version of this figure is available in the online journal.)

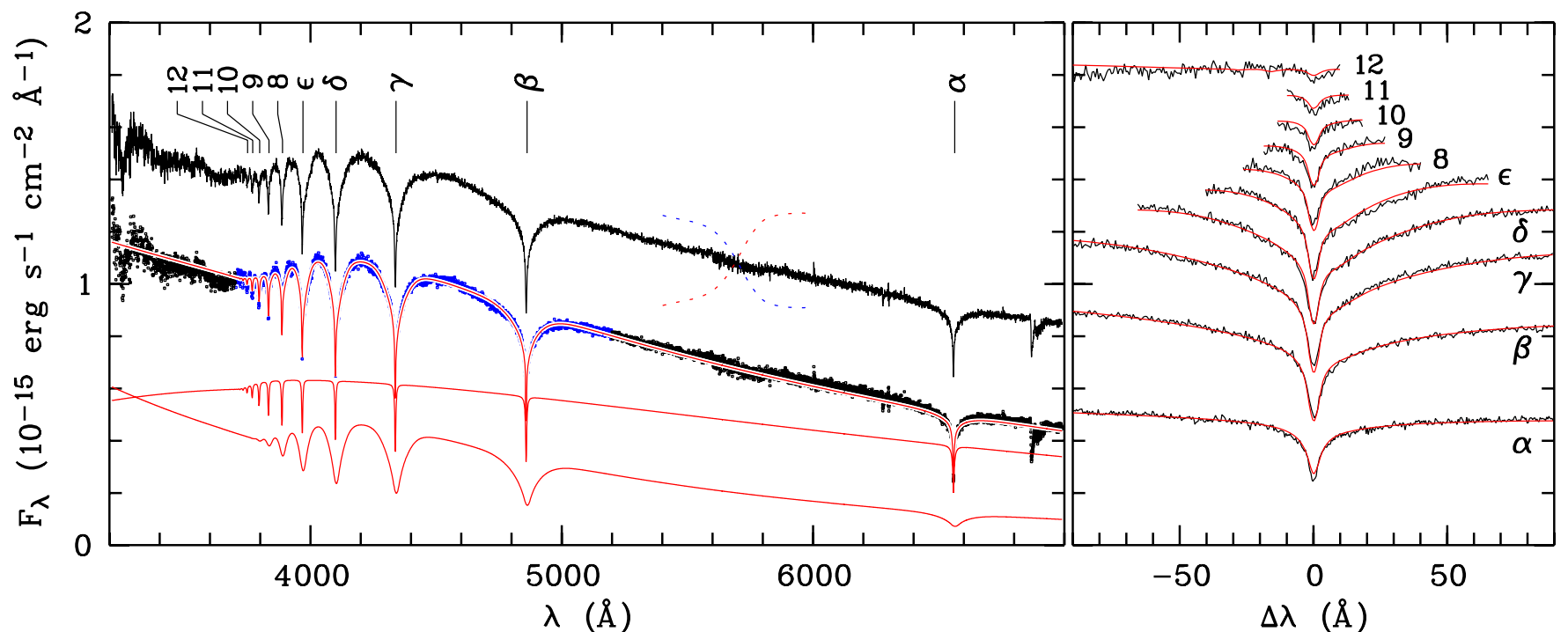

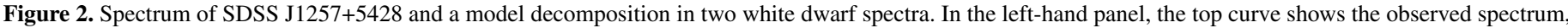

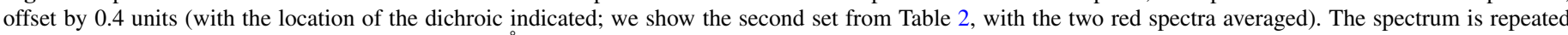

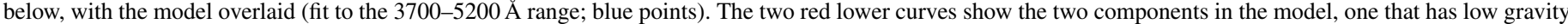

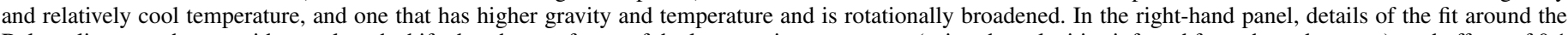

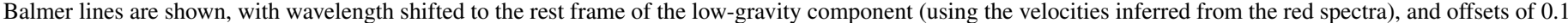
in flux added between all profiles beyond $\mathrm{H} \beta$.

(A color version of this figure is available in the online journal.)

\subsection{Model Fitting}

The above qualitative impressions are born out by fits with model atmospheres. For these fits, we modeled the blue spectra, in the range of 3700-5200 $\AA$ (i.e., the range showing lines), with a combination of two hydrogen model atmospheres, taken from a set kindly provided by $\mathrm{D}$. Koester. ${ }^{7}$

\footnotetext{
7 This is Koester's most recent version of grid of white dwarf spectra. The basic model is described in Koester et al. (2009b) and the latest version includes the improved treatment of pressure broadening by Tremblay \& Bergeron (2009). In detail, the grid spans $6000 \mathrm{~K}<T_{\text {eff }}<30000 \mathrm{~K}$ in steps of $250-1000 \mathrm{~K}$ at low and high temperature and $6.0<\log g<9.5$ in steps of 0.25 dex.
}

We scanned a grid in effective temperature and surface gravity for each component, as well as a set of rotational velocities $0<v_{r} \sin i<2000 \mathrm{~km} \mathrm{~s}^{-1}$ for the higher-gravity component (in steps of $200 \mathrm{~km} \mathrm{~s}^{-1}$, we convolved the model using the analytical profile of Gray 2005, with a limb darkening coefficient of $\left.0.3^{8}\right)$. For the lower-gravity component, we took the smearing due to changing orbital velocity into account (using the orbit of Marsh et al. 2010; the largest effect is $80 \mathrm{~km} \mathrm{~s}^{-1}$, substantially smaller than our resolution; the motion of the high-gravity component during an integration is negligible).

\footnotetext{
8 The limb darkening coefficient is inferred from model specific intensities used previously to fit spectra of pulsating white dwarfs (Clemens et al. 2000). We ignored the variation in limb darkening over the lines.
} 
Table 3

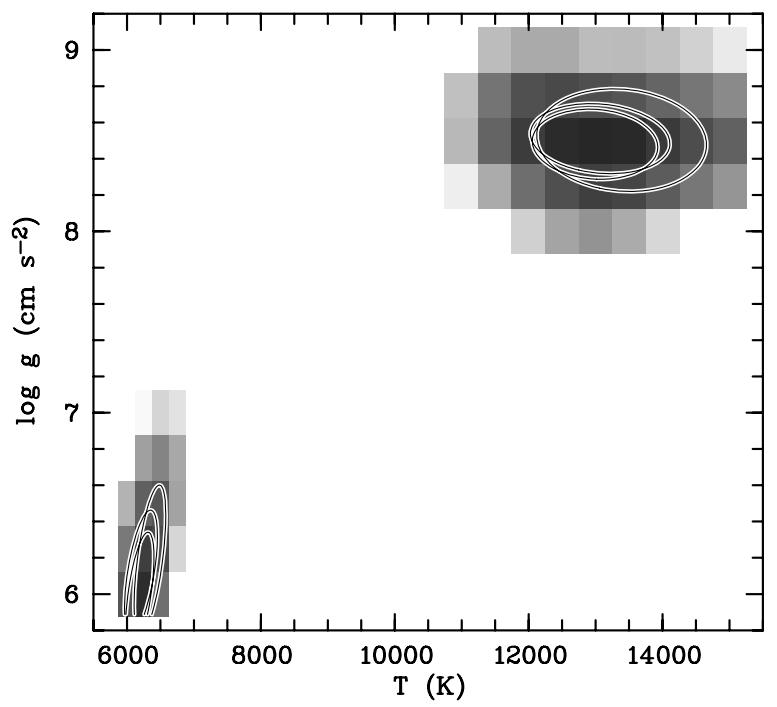

Figure 3. Constraints on the properties of the two white dwarfs in SDSS $1257+5428$. The gray scale shows summed $\chi^{2}$ values for the fits to the three blue spectra (see Figure 2), within the range of $\chi_{\min }^{2} \simeq 12000$ $\left(\chi_{\text {red,min }}^{2}=1.7\right.$; darkest $)$ to $\chi^{2}>14500\left(\chi_{\text {red }}^{2}>2.0\right.$; white $)$. For each white dwarf, at each grid point, we minimized over the parameters of the other white dwarf, the broadening of the massive white dwarf, the two radial velocities, the flux ratio, and the continuum normalization. The three contours for each white dwarf show the results of the fits to the three individual blue spectra; they are drawn at $\chi_{\min }^{2}+100\left(\chi_{\text {red,min }}^{2}+0.04\right)$, a level that we believe gives a conservative estimate of the uncertainties even in the presence of systematic residuals in the fits (see the text).

We accounted for the spectral resolution by convolving all models with a truncated Gaussian (with width and truncation matching the seeing and slit width, respectively) and resampling them at the observed wavelengths. At each grid point, we fitted for the two best-fit velocities, the flux ratio, and a normalization (which, to account for possible errors in our flux calibration, we allowed to depend quadratically on wavelength).

We show the best fit to one of the three sets of spectra in Figure 2. One sees that, overall, the fit is good, also outside the fitted range (indeed, it reproduces the spectral slope remarkably well). The fits yield a reasonably well-defined minimum, with a cool, $T_{1} \simeq 6250 \mathrm{~K}$, low gravity, $\log g_{1} \simeq 6.0$ primary and a hotter, $T_{2} \simeq 13000 \mathrm{~K}$, more massive, $\log g_{2} \simeq 8.5$ companion that is rotating rapidly, $v \sin i \simeq 800 \mathrm{~km} \mathrm{~s}^{-1}$ (see Figure 3 and Table 3).

While the fits are good overall, some details are not matched: the highest Balmer lines are predicted slightly too weak, and some line cores are not reproduced accurately ( $\mathrm{H} \alpha$ being not deep enough, and $\mathrm{H} \gamma$ and $\mathrm{H} 8$ too deep). These effects might indicate that the true gravity of the primary is slightly lower than that in our fit (which is at the lower boundary of our grid). Furthermore, the red wings of some of the lines ( $\mathrm{H} \epsilon$ and $\mathrm{H} 8$ in particular) are poorly matched. These differences may be due to inaccuracies in the flux calibration. Indeed, the spectra of Marsh et al. (2010) show discrepancies that are qualitatively similar but differ in detail. To resolve, this would likely require more careful spectrophotometry, e.g., using a wider slit and using a flux standards that is itself a white dwarf, of similar temperature, that can be fit with the same set of models.

Because of the above problems, even though the fit is hugely superior to one using only a single white dwarf (cf. Figure 5 of Badenes et al. 2009), it is still not formally acceptable; for the
Spectroscopic Properties of the White Dwarfs in SDSS 1257+5428

\begin{tabular}{lcc}
\hline \hline \multicolumn{1}{c}{ Property } & Phot. Primary & Phot. Secondary \\
\hline Fit parameters & & \\
$T_{\text {eff }}(\mathrm{K})$ & $6250 \pm 250$ & $13000 \pm 800$ \\
$\log g\left(\mathrm{~cm} \mathrm{~s}^{-2}\right)$ & $6.0 \pm 0.3$ & $8.5 \pm 0.2$ \\
$v_{r} \sin i\left(\mathrm{~km} \mathrm{~s}^{-1}\right)$ & $<100$ & $800 \pm 300$ \\
$R_{2} / R_{1}$ & $0.21 \pm 0.03$ \\
Derived parameters & & \\
$M\left(M_{\odot}\right)$ & $0.15 \pm 0.05$ & $0.92 \pm 0.13$ \\
$R\left(R_{\odot}\right)$ & $0.042 \pm 0.008$ & $0.0089 \pm 0.0012$ \\
\hline
\end{tabular}

Notes. The parameters are for a model in which the binary is composed of two white dwarfs, with the secondary rotating rapidly. The limit to the rotation of the primary is derived separately from $\mathrm{H} \alpha$; at the limit, the fit to the core is obviously worse. Note that the model fit is not formally acceptable (see the text); the quoted uncertainties are what we believe are conservative estimates.

spectra taken through the $1^{\prime \prime}$ slit, we find $\chi_{\text {red }}^{2} \simeq 1.7$ (for 2408 data points and 13 parameters; $\chi_{\text {red }}^{2} \simeq 1.5$ for the single, less well-exposed spectrum taken through a $00^{\prime \prime} 7$ slit). While the fit is internally consistent, in that the three blue spectra give the same best-fit grid point (see Figure 3), and that, e.g., fits to $\mathrm{H} \alpha$ and to higher Balmer lines give roughly consistent results (for the latter, though, there is a much larger covariance with the properties of the high-gravity component), we cannot know what offsets are caused by the systematic differences. We give what we believe are conservative error estimates in Table 3 , derived by finding the change in the parameter of interest for which $\chi^{2}$ increases by 100 (while minimizing over all other parameters, i.e., our uncertainties are 10 times the formal $1 \sigma$ errors). For the lowmass white dwarf, the fits to the line cores are noticeably worse at our quoted limits; for the massive white dwarf, the decrease in the quality of the fits is less obvious visually, as the deviations occur over larger wavelength ranges.

Finally, we searched for the presence of He I $5876 \AA$ (see Section 2). Inspecting the spectrum, we determined that a $2 \%$ depression would be clearly noticeable. If an absorption feature existed at the limit of instrumental resolution, then the equivalent width limit would be $0.02 \times 3 \AA=0.06 \AA$. If the feature was broadened by $800 \mathrm{~km} \mathrm{~s}^{-1}$, corresponding to $\Delta \lambda \sim 15 \AA$, the equivalent width limit is $0.3 \AA$.

\subsection{Comparison}

Comparing our inferred parameters with those given by Marsh et al. (2010), one finds qualitative agreement, in inferring a cool, low-gravity and a hotter, high-gravity component, but what would appear significantly different parameters (Marsh et al. 2010 find $T_{\text {eff }, 1}=7200 \pm 350 \mathrm{~K}, \log g_{1}=6.85 \pm 0.10$, $T_{\text {eff }, 2} \simeq 9800 \pm 1000 \mathrm{~K}$, and $\log g_{2} \simeq 9.0 \pm 0.4$ ). It appears that the most important difference is that in $T_{\text {eff, } 2}$ : if we fix this temperature, the remaining parameters become comparable to those of Marsh et al. (2010), though the fit is much worse $\left(\chi_{\text {red }}^{2} \simeq 3.2\right)$. The same does not hold if we fix other parameters (e.g., for $T_{\text {eff }, 1}=7250$, the fit quality is similarly poor for a large range in $\left.T_{\text {eff, } 2}\right)$. The underlying problem may be that the Balmer lines have similar strength at $10,000 \mathrm{~K}$ and $13,000 \mathrm{~K}$. This leads to a degeneracy for normalized spectra such as those presented by Marsh et al. (2010). Usually, such degeneracies can be broken by optical photometry, but here one can change the temperature of the other component to compensate. Fortunately, the ultraviolet data presented by Marsh et al. (2010) show conclusively that the high-temperature solution is the correct one. 
Turning now to the velocities, like Marsh et al. (2010), we find that our fits yield precise measurements of the motion of the photometric primary, in both our blue and red spectra, but not of that of the secondary. For the primary, for the blue spectra the accuracy is limited by flexure in the spectrograph, which we appear to be able to correct only partially using the $5577 \AA$ sky line. In Table 2, we list the barycentric velocities inferred from $\mathrm{H} \alpha$; these are consistent with the radial-velocity amplitude of $330 \mathrm{~km} \mathrm{~s}^{-1}$ and the systemic velocity of $28.9 \mathrm{~km} \mathrm{~s}^{-1}$ found by Badenes et al. (2009) and Marsh et al. (2010). For the secondary, we find that while for every choice of parameters, its velocity can be measured in the blue and is consistently found at a positive offset from the primary (as expected given the orbital phases and its larger gravitational redshift), the precise values strongly depend on the temperature of the primary, with changes of $250 \mathrm{~K}$ leading to velocity differences of $\sim 50,40$, and $30 \mathrm{~km} \mathrm{~s}^{-1}$ for the three different spectra. Thus, we cannot measure its motion.

\subsection{Inference}

From the best-fit model parameters for the secondary (Table 3) and using the cooling models of Wood (1992), we deduce the following: $M_{2}=0.92 \pm 0.13 M_{\odot}$ and $R_{2}=$ $0.0089 \pm 0.0012 R_{\odot}$, and a cooling age of $\tau_{c}=0.7 \pm 0.3 \mathrm{Gyr}$.

For the primary, we start with the normalization in the spectral fits, which yields the ratio of emitting areas for the two components, and thus $R_{2} / R_{1}=0.21 \pm 0.03$. Combined with the estimate for $R_{2}$, one finds $R_{1}=0.042 \pm 0.008 R_{\odot}$. The spectral fits yield $\log g_{1}=6.0 \pm 0.3$ (but more about this below). The mass of the primary can be directly computed from $\log g_{1}$ and the radius, $R_{1}$. The mean value is $0.07 M_{\odot}$; however, due to the large error in $\log g$, the distribution of $M_{1}$ has a long tail with a variance comparable to that of the mean.

Thus, we cannot determine the mass directly, but we can compare the parameters with those for other low-mass white dwarfs. The first were seen as companions to millisecond pulsars (for a review, see van Kerkwijk et al. 2005) and more recently field objects were found via SDSS (Kilic et al. 2007). Some notable objects are SDSS J0917+46 $(\log g=5.48 \pm 0.03$, $T_{\text {eff }}=11288 \pm 72 \mathrm{~K}$; Kilic et al. 2007); NLTT $11748(\log g=$ $6.20 \pm 0.05, T_{\text {eff }}=8540 \pm 50 \mathrm{~K}$; Kawka \& Vennes 2009); and LP 400-22 $\left(\log g=6.35 \pm 0.05, T_{\text {eff }}=11170 \pm 90 \mathrm{~K}\right.$; Vennes et al. 2009). The latter is also notable because it has a high space motion, even though its companion is another white dwarf.

From evolutionary considerations, the true mass of the primary in SDSS $1257+5428$ is likely higher than the above estimate of $0.07 M_{\odot}$ from $\log g$. This is because to form a heliumcore white dwarf, the progenitor must have evolved off the main sequence within a Hubble time and thus have had a mass of $\gtrsim 0.8 M_{\odot}$. Such a progenitor would have had a minimum core mass of $\sim 0.12-0.18 M_{\odot}$, with the precise value depending on, e.g., metallicity (see Pols et al. 1998). Thus, one infers a true $\log g_{1} \gtrsim 6.3$. This is on the upper limit of our measurement, but we note that the model atmospheres appear not to be entirely reliable in this low-gravity regime. Specifically, models from different groups gave results different by 0.4 dex for the low gravity, $\log g \simeq 6.5$ companion to PSR J1012+5307 (see van Kerkwijk et al. 2005).

An upper limit to the mass of the primary can be derived by combining the inferred mass of the secondary with the orbital information (see Figure 4). Rewriting Equation (1), one finds $M_{2}=M_{1}\left[\left(M_{1} \sin ^{3} i / \mathcal{M}_{2}\right)^{1 / 2}-1\right]<0.15 \pm 0.08 M_{\odot}$ for $M_{2}=0.92 \pm 0.13 M_{\odot}$.

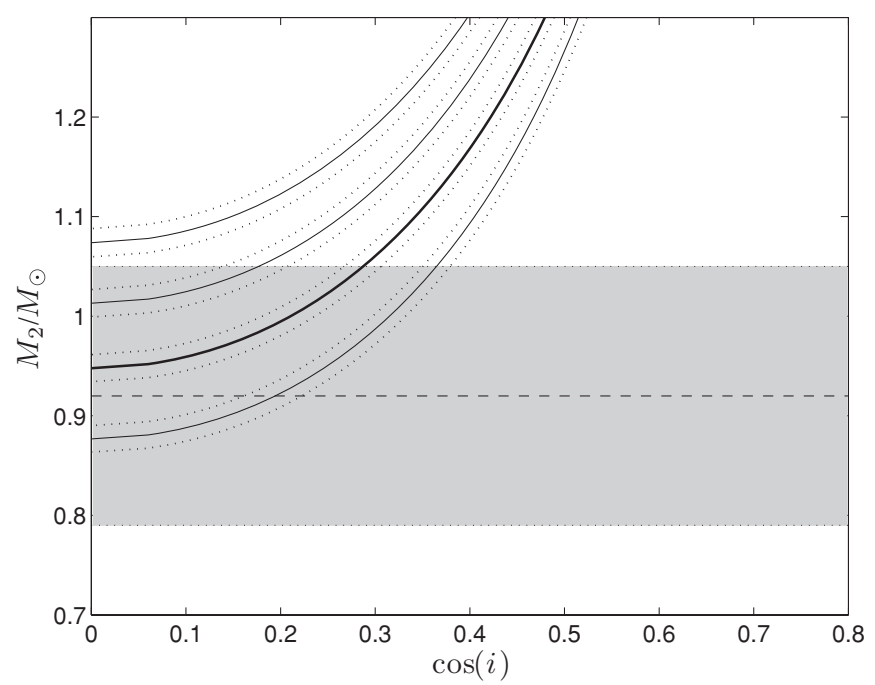

Figure 4. Mass of the photometric secondary as a function of $\cos i$ where $i$ is the angle between the orbital angular momentum and the line of sight (from bottom to top, $\left.M_{1}=0.10,0.15,0.20,0.25 M_{\odot}\right)$. Here, we adopt the radial-velocity amplitude for the primary from Marsh et al. (2010), $K_{1}=330 \pm 2 \mathrm{~km} \mathrm{~s}^{-1}$. The pair of dotted lines represent the uncertainty in $M_{2}$ (for a given value of $M_{1}$ ) arising solely from the $1.8 \%$ uncertainty in the mass function, $\mathcal{M}_{2}$. The nominal value of $M_{1}=0.15 M_{\odot}$ is shown by the thick line. The bounds on the secondary mass $M_{2}$ from spectral fitting are shown by the horizontal dotted lines. The totality of the data argue that $M_{1} \lesssim 0.2 M_{\odot}$.

Overall, combining all constraints, we conclude that the primary is a helium-core white dwarf with a mass close to the minimum possible. Below, we will take $M_{1} \simeq 0.15 \pm 0.03 M_{\odot}$.

\section{SDSS 1257+5428, A DOUBLE DEGENERATE: RAMIFICATIONS}

Above, we established that SDSS $1257+5428$ is not a neutronstar binary but a bona fide double degenerate system with parameters given in Table 3. Here, we explore the consequences of this conclusion.

\subsection{Distance and Demography}

The distance to SDSS $1257+5428$ is increased because the flux of SDSS $1257+5428$ is now dominated by a lower mass white dwarf (with a larger radius and hence a larger angular diameter relative to that in the Badenes et al. 2009 model). From our fit (see Table 3), we infer a distance to SDSS $1257+5428$ of about $140 \mathrm{pc}$ (instead of $50 \mathrm{pc}$ ).

With the new properties and distance, is SDSS $1257+5428$ still unique? There exist 129 systems (single white dwarfs, white dwarf binaries, and double degenerate binary systems) within $20 \mathrm{pc}$ (Sion et al. 2009). Holberg et al. (2008) find seven double degenerate systems within $20 \mathrm{pc}$, consistent with the range of 1.7\%-19\% (95\% confidence level) of double degenerates among white dwarfs estimated by Maxted \& Marsh (1999). Thus, within $140 \mathrm{pc}$ one expects to find about 2400 double degenerates (or 700-9000 using the above range).

Furthermore, the systemic radial velocity of SDSS $1257+5428$ is now $\gamma_{1}-\gamma_{E}(1)$, where the former is the mean radial velocity of the primary (see Section 3) and $\gamma_{E}(1)$ is the gravitational redshift for the primary. With the revised mass, the redshift is small, $\gamma_{E}(1)=2$ to $5 \mathrm{~km} \mathrm{~s}^{-1}$ (for $M_{1}=0.1-0.2 M_{\odot}$ ). Thus, the three-dimensional velocity of SDSS $1257+5428$ is quite consistent with that of other double degenerates. We conclude that SDSS $1257+5428$ is not a rare system. 


\subsection{Coalescence Timescale}

Double degenerates, especially in compact binaries, lose energy by gravitational radiation and eventually coalesce. The time for the orbital period to halve is (Lang 1980, p. 600):

$$
\begin{aligned}
\frac{1}{P_{\mathrm{b}}} \frac{d P_{\mathrm{b}}}{d t} & =-\frac{96}{5} \frac{G}{c^{5}} \frac{\mu M^{2}}{a^{4}} \\
& =\left(\frac{1}{2.8 \times 10^{7} \mathrm{yr}}\right)\left(\frac{M}{M_{\odot}}\right)^{2 / 3}\left(\frac{\mu}{M_{\odot}}\right)\left(\frac{1 \mathrm{hr}}{P_{\mathrm{b}}}\right)^{8 / 3}
\end{aligned}
$$

where $M=M_{1}+M_{2}$ and $\mu=M_{1} M_{2} / M$. Integrating this equation to zero period yields a coalescence timescale of

$$
\tau_{\mathrm{GW}}=1.05 \times 10^{7} \mathrm{yr}\left(\frac{M}{M_{\odot}}\right)^{-2 / 3}\left(\frac{\mu}{M_{\odot}}\right)^{-1}\left(\frac{P_{\mathrm{b}}}{1 \mathrm{hr}}\right)^{8 / 3} .
$$

For $M_{1}=0.15 \pm 0.05 M_{\odot}$ and $M_{2}=0.92 \pm 0.13 M_{\odot}$, we find $\tau_{\mathrm{GW}}=4.4 \mathrm{Gyr}$. At the one percentile level, the range is 2.5-15 Gyr. (For the total duration, one needs to add the cooling time from Section 5.3; Phinney 1991.)

\subsection{Merger Rate}

Thompson et al. (2009), accepting the masses of and distance to SDSS $1257+5428$ of Badenes et al. (2009), found a very large rate of coalescence of SDSS 1257+5428-like systems-comparable to that of Ia supernovae. The revision of the distance, masses, and scale height reduces the coalescence rate by a large factor: using a scale height of $0.5 \mathrm{kpc}$ typical for older stars and white dwarfs instead of $4 \mathrm{kpc}$ for neutron-star binaries; a distance of $140 \mathrm{pc}$ to SDSS $1257+5428$ instead of $50 \mathrm{pc}$ (Section 6.1); and a lifetime of $\sim 5 \mathrm{Gyr}$ instead of $2 \mathrm{Gyr}$ (Section 6.2), our estimate of the Galactic coalescence rate is a factor $4 / 0.5 \times(140 / 50)^{3} \times 5 / 2$ smaller, or

$$
\gamma_{\mathrm{MW}} \sim 1 \times 10^{-6} \mathrm{~N} \mathrm{yr}^{-1},
$$

where $N=1$ is the number of such systems detected (and consequently suffers from severe Poisson uncertainty).

The rate given in Equation (4) is not alarming (corresponding to a mean time between events of $\sim 1 \mathrm{Myr}$ ). Furthermore, given that $M_{1}+M_{2}$ does not incontrovertibly exceed the Chandrasekhar limit, the outcome of this coalescence is (conservatively) not a type Ia supernova explosion, but rather the formation a hydrogen-poor giant or an AM CVn system (see the discussion in Kilic et al. 2010). The rate given in Equation (4) is small enough that, even if the combined masses were to exceed the Chandrasekhar limit, SDSS 1257+5428-like systems are not major contributors to the supernovae Ia rate.

\subsection{Broadening: Rotation?}

As noted in Section 5, we are forced to invoke broadening for the Balmer series of the secondary and modeled this as rotation. We consider this simplest possibility first. Our inferred rotation rate is $v_{r} \sin i=800 \pm 300 \mathrm{~km} \mathrm{~s}^{-1}$ (see Table 3), where $i$ is the inclination between the rotation axis of the secondary and the line of sight. We will assume that the spin of the secondary is aligned with the angular momentum of the binary.

For the entire range of plausible values for $M_{2}$ and the bounds for $M_{1}$, we find that $\cos i<0.4$ and thus $\sin i>0.84$ (see Figure 4). In view of the errors on $v \sin i$, we will approximate $\sin i \approx 1$. Using our derived value for the radius of the secondary, $R_{2}=0.0089 \pm 0.0012 R_{\odot}$ (see Section 5), we find that the rotational period, $P \simeq 50_{-15}^{+30} \mathrm{~s}$, where the $30 \%$ uncertainty in $v \sin i$ dominates the uncertainty. We will adopt a round figure of $60 \mathrm{~s}$ for the rotation period of the secondary and write corresponding angular frequency as $\omega=2 \pi / P$.

\subsubsection{Spin-up by Accretion}

The simplest possibility that comes to our mind as an explanation for rapid rotation of the secondary is spin up by accretion of matter. In order to lead to significant spin-up, this should happen in a relatively long-lived phase, and hence it seems unlikely the system was brought to its present state by a common-envelope phase (furthermore, to survive a commonenvelope phase, the system would have to have been relatively wide, and one would expect a somewhat more massive heliumcore white dwarf). Instead, it seems most likely the evolution would be similar to what is invoked to explain the millisecond periods of neutron stars in binaries for both long and short periods $\left(P_{\mathrm{b}}>1\right.$ day, Webbink et al. 1983; $P_{\mathrm{b}}<1$ day, Pylyser \& Savonije 1988). Depending on the evolutionary status of the mass donor and the orbital separation, mass transfer to a higher mass accretor (in this case the white dwarf photometric secondary) is driven by nuclear evolution, loss of angular momentum via stellar winds ("magnetic braking") or gravitational wave radiation. Pylyser \& Savonije (1988) find that the typical accretion rate for short-period binaries is $\sim 10^{-9} M_{\odot} \mathrm{yr}^{-1}$, which, for a white-dwarf accretor, correspond to an accretion luminosity $G M_{2} \dot{M} / R_{2} \simeq 10^{34} \mathrm{erg} \mathrm{s}^{-1}$.

To estimate the mass required to spin up the white dwarf, we first consider the case where the accreting white dwarf is unmagnetized. In this case, the accreted matter has the specific orbital angular momentum appropriate for the radius of the white dwarf, or $\Omega_{2} R_{2}^{2}$, where the orbital angular frequency $\Omega_{2}=\left(G M_{2} / R_{2}{ }^{3}\right)^{1 / 2}$ corresponds to a period of $\sim 7 \mathrm{~s}$ (for $\left.M_{2} \approx 1 M_{\odot}\right)$. Thus, for an accreted mass $\Delta M$, the total amount of accreted angular momentum is $\Delta M \Omega_{2} R_{2}{ }^{2}$. Equating this to the current angular momentum, $k_{2} M_{2} R_{2}^{2} \omega$, with $k_{2} \simeq 0.143$ appropriate for an $n=1.5$ polytrope (Brooker \& Olle 1955), we infer an accreted fractional mass,

$$
\frac{\Delta M}{M_{2}}=k_{2} \frac{\omega}{\Omega_{2}}=0.017 .
$$

It is empirically known that accreting white dwarfs in CVs do not rotate rapidly (e.g., Gänsicke et al. 2005), and one deduces that the accreted angular momentum is lost. Presumably, this is lost in nova explosions, but this requires that for most CVs there is poor angular momentum coupling between the accreted matter and the core (Livio \& Pringle 1998). If so, each nova cycle, in effect, leaves both the mass and the spin state of the accreting white dwarf unaffected.

For the low accretion rates one expects for the progenitor of SDSS 1257+5428, nova explosions will occur (Fujimoto 1982). These happen when the accreted mass exceeds a certain "ignition" value, which depends on the mass of the white dwarf. According to Townsley \& Bildsten (2004), the ignition masses are $M_{\mathrm{ig}} \approx(6,3,2) \times 10^{-4} M_{\odot}$ for $(0.6,1.0,1.2) M_{\odot}$ white dwarfs. Thus, assuming that the white dwarf accumulated no more than $M_{\text {ig }}$ following the very last nova explosion, the spin period can be no faster than $\sim\left(2 \pi / \Omega_{2}\right)\left(k_{2} M_{2} / M_{\mathrm{ig}}\right) \simeq 1 \mathrm{hr}$ (for $\left.M_{2} \simeq 1 M_{\odot}\right)$.

The minimum amount of accreted matter can be reduced for the case when the accreting white dwarf is magnetized. In this case, the accreting matter has the specific angular momentum 
$\sqrt{G M r_{A}}$, where $r_{A}$ is the Alfvén radius. This matter has more specific angular momentum compared to the previous case by the factor $\sqrt{r_{A} / R_{2}}$, which, once the white dwarf is spun up such that $r_{A}$ approaches the corotation radius, scales as $\propto\left(\Omega_{2} / \omega\right)^{1 / 3}$ via Kepler's third law. Hence, in this case the minimum accreted matter is reduced to

$$
\frac{\Delta M}{M_{2}}=k_{2} \frac{\omega}{\Omega_{2}}\left(\frac{\omega}{\Omega_{2}}\right)^{1 / 3}=0.008
$$

However, even this mass exceeds the ignition masses by more than an order of magnitude. Although it may be possible to find evolutionary histories in which mass transfer is much more rapid, or that have, e.g., an initial phase with rapid mass transfer, we feel this would be somewhat contrived. Thus, we reject the spin-up by accretion model, at least for the case where the white dwarf is not magnetized or magnetized sufficiently weakly that the accreted envelope does not couple to the core.

\subsubsection{Intermediate Polar Model}

There exists a class of CVs called DQ Her stars or intermediate polars whose white dwarfs are found to be spinning quite rapidly (Patterson 1994). These consist of a strongly magnetized white dwarf accreting matter from the companion. The most famous example is AE Aquarii with a spin period of $32 \mathrm{~s}$. A number of intermediate polars have spin periods of about a minute. ${ }^{9}$ Motivated by the similarity of the spin periods of intermediate polars and that inferred for SDSS $1257+5428$, we explore an intermediate polar model.

Intermediate polars undergo nova explosions (e.g., GK Per $=$ Nova Persei 1901; DQ Her = Nova Herculis 1934; see Livio 1983). (Novae also have been seen from polars, in which the white dwarf rotation is magnetically locked to the orbit, e.g., V1500 Cyg = Nova Cygni 1975; Chlebowski \& Kaluzny 1988.) Thus, the rapid rotation seen in intermediate polars, despite the novae, shows that the white dwarfs manage to retain the accreted angular momentum. We infer that the strong magnetic field ensures rapid coupling between the accreted matter and the rest of the white dwarf. Subsequent novae explosions merely result in ejection of the matter and a very modest fractional loss in angular momentum, $\frac{2}{3} M_{\mathrm{ig}} /\left(k_{2} M_{2}\right)$ (where we assumed $I_{\mathrm{ej}}=\frac{2}{3} M_{\mathrm{ig}} R_{2}^{2}$ for the ejected shell).

Following the accretion of the minimum mass, the white dwarf will not be spun up any further, but reach an equilibrium spin period, given by (Ghosh \& Lamb 1979)

$$
P_{\text {eq }}=6.3 \times B_{4}^{6 / 7} L_{34}^{-3 / 7}\left(\frac{M}{M_{\odot}}\right)\left(\frac{R}{5 \times 10^{8} \mathrm{~cm}}\right) \mathrm{s} .
$$

Here, $B=10^{4} B_{4} \mathrm{G}$ is the dipole field of the white dwarf and $L=10^{34} L_{34} \mathrm{erg} \mathrm{s}^{-1}$ is the accretion luminosity during the accretion phase. With $M=0.9 M_{\odot}$ and $R=0.009 R_{\odot} \simeq$ $6 \times 10^{8} \mathrm{~cm}$, we see that a modest (dipolar) field of $1.3 \times 10^{5} \mathrm{G}$ is sufficient to account for the inferred period of $\sim 60 \mathrm{~s}$.

The dynamical equation for the spin-up is given by

$$
\frac{\dot{\omega}}{2 \pi} \approx 3 \times 10^{-16} \mathrm{~Hz} \mathrm{~s}^{-1} L_{34}^{3 / 7} \mu_{30}^{2 / 7} I_{50}^{-1}\left(\frac{M}{M_{\odot}}\right)\left(\frac{R}{5 \times 10^{8} \mathrm{~cm}}\right)^{6 / 7},
$$

\footnotetext{
9 See http://asd.gsfc.nasa.gov/Koji.Mukai/iphome/iphome.html for a catalog of intermediate polars.
}

where $\mu=B R^{3}$ is the magnetic moment and the normalization is that for a $10^{4} \mathrm{G}$ dipole field and radius appropriate for a solar mass white dwarf. The white dwarf would be spun up (assuming a suitable accretion rate) in less than two million years.

We conclude that the intermediate polar model is a good explanation for the origin of spin in SDSS 1257+5428. As an aside, we make the following observation. The fastest intermediate polars have periods of about a minute. This then empirically suggests that a magnetic field strength of $\sim 10^{5} \mathrm{G}$ is the minimum field strength required to rapidly couple the white dwarf and the accreted envelope.

\subsection{Broadening: Magnetism?}

Above, we assumed that the observed broadening in the high-gravity spectrum is due to rotation. An entirely different possibility is that it is magnetic. In a strong magnetic field, the Balmer series are split into three components: two senses of circular polarization and an unpolarized component. To first order, the circular polarization components are shifted by half the electron cyclotron energy, or $0.0057 \mathrm{eV}\left(B / 10^{6} \mathrm{G}\right)$, on each side of the unperturbed unpolarized component. At second order, all lines will shift bluewards, with the shift substantially stronger for transitions to higher excited states.

For the field strength of $10^{5} \mathrm{G}$ inferred above, the total expected spread is $0.0011 \mathrm{eV}$, which corresponds to a "velocity width" of about $200 \mathrm{~km} \mathrm{~s}^{-1}$ at $\mathrm{H} \alpha$ (and smaller proportionally with $\lambda^{2}$ for the higher Balmer lines). This in itself will not have a noticeable effect on the model fits.

However, a possibility is that the white dwarf is not rotating rapidly at all, but has a stronger field strength, say approaching $10^{6} \mathrm{G}$. In this case, the observed broadening could be almost entirely due to Zeeman splitting (e.g., as in WD 0637+477; Schmidt et al. 1992). Indeed, this might be the cause for the relatively poor spectral fits. We looked for the expected decrease in broadening and increase in blueshift for the higher Balmer lines, as well as for possible evidence for split line cores (which might occur if the field is sufficiently uniform), but we were unable to conclusively accept or reject the hypothesis that the Balmer lines are broadened by Zeeman splitting.

\section{TESTING THE MODEL}

We found that SDSS $1257+5428$ is composed of two white dwarfs, one relatively cool one with low gravity, and another, hotter one with higher gravity whose lines are substantially broadened. A question that is left is what is the cause for the broadening, rapid rotation with a period of about a minute, or a strong, $\sim 10^{6} \mathrm{G}$, strength field. The clearest way to distinguish the two models would be by spectropolarimetry. For the case of rapid rotation, this would also allow one to test whether, as we contended, such rapid rotation requires the presence of a weaker field, of $\sim 10^{5} \mathrm{G}$, similar to the fields inferred for intermediate polars. Rapid photometry (and spectrometry) could perhaps even reveal the rotation period. With $\log g=9.5$ and $T_{\text {eff }}=13,000 \mathrm{~K}$, the secondary white dwarf lies close to the $\mathrm{ZZ}$ Ceti strip (Gianninas et al. 2006) and so a search for pulsations could be quite productive.

Apart from its intrinsic interest, SDSS 1257+5428 may also become a useful test case for white dwarf models. With sufficient sensitivity and orbital coverage, it should be possible to measure velocity curves for both components (i.e., derive $\left.M_{2} / M_{1}\right)$ and determine the difference in gravitational redshift, (i.e., $\left.(M / R)_{2}-(M / R)_{1}\right)$. Further model-atmosphere fits would 
yield much more accurate temperatures and gravities (i.e., $\left.\left(M / R^{2}\right)_{1,2}\right)$ and a precise ratio of the emitting areas (i.e., $\left.\left(R_{2} / R_{1}\right)^{2}\right)$. Combined with the theoretical mass-radius relation, the system is thus strongly overconstrained and can be used to test various assumptions. This would be especially valuable for the low-mass companion, since, as noted in Section 5.3, for the very low mass white dwarfs the atmosphere and mass-radius relation are currently not as securely established as they are for the case of regular white dwarfs.

We thank E. S. Phinney, G. Nelemans, C. Badenes, and L. Bildsten for discussions. LRIS has been upgraded since its commissioning and as a result is perhaps now the most efficient single object optical spectrometer. We thank the teams that made these improvements possible (J. McCarthy and C. Steidel, leaders for LRIS-Blue upgrade; C. Rokosi, leader for the LRIS-Red upgrade; and J. Miller and D. Phillips, leaders for the ADC sub-system). We are grateful to the staff of the WM Keck Observatory for their excellent service and to the librarians who maintain the ADS and Simbad databases.

\section{REFERENCES}

Abazajian, K. N., et al. 2009, ApJS, 182, 543

Badenes, C., Mullally, F., Thompson, S. E., \& Lupton, R. H. 2009, ApJ, 707, 971

Bèland, S., Boulade, O., \& Davidge, T. 1988, Bulletin d'information du telescope Canada-France-Hawaii, 19, 16

Bergeron, P., Saffer, R. A., \& Liebert, J. 1992, ApJ, 394, 228

Brisken, W. F., Benson, J. M., Goss, W. M., \& Thorsett, S. E. 2002, ApJ, 571, 906

Brooker, R. A., \& Olle, T. W. 1955, MNRAS, 115, 101

Chlebowski, T., \& Kaluzny, J. 1988, Acta Astron., 38, 329

Clemens, J. C., van Kerkwijk, M. H., \& Wu, Y. 2000, MNRAS, 314, 220

Cordes, J. M., \& Chernoff, D. F. 1997, ApJ, 482, 971

Deller, A. T., Tingay, S. J., Bailes, M., \& Reynolds, J. E. 2009, ApJ, 701, 1243

Deller, A. T., Verbiest, J. P. W., Tingay, S. J., \& Bailes, M. 2008, ApJ, 685, L67

Dodson, R., Legge, D., Reynolds, J. E., \& McCulloch, P. M. 2003, ApJ, 596, 1137

ESO Users Manual 1993, The Messenger, 73, 47

Faherty, J., Walter, F. M., \& Anderson, J. 2007, Ap\&SS, 308, 225

Falcon, R. E., Winget, D. E., Montgomery, M. H., \& Williams, K. A. 2010, ApJ, 712,585

Fujimoto, M. Y. 1982, ApJ, 257, 767

Gänsicke, B. T., Szkody, P., Howell, S. B., \& Sion, E. M. 2005, ApJ, 629, 451

Ghosh, P., \& Lamb, F. K. 1979, ApJ, 234, 296
Gianninas, A., Bergeron, P., \& Fontaine, G. 2006, AJ, 132, 831

Gray, D. F. 2005, The Observation and Analysis of Stellar Photospheres (Cambridge: Cambridge Univ. Press)

Hamada, T., \& Salpeter, E. E. 1961, ApJ, 134, 683

Holberg, J. B., Sion, E. M., Oswalt, T., McCook, G. P., Foran, S., \& Subasavage, J. P. 2008, AJ, 135, 1225

Horne, K. 1986, PASP, 98, 609

Kaplan, D. L., \& van Kerkwijk, M. H. 2009, ApJ, 705, 798

Kawka, A., \& Vennes, S. 2009, A\&A, 506, L25

Kilic, M., Allende Prieto, C., Brown, W. R., \& Koester, D. 2007, ApJ, 660, 1451

Kilic, M., Brown, W. R., Allende Prieto, C., Kenyon, S. J., \& Panei, J. A. 2010, ApJ, 716, 122

Koester, D., Kepler, S. O., Kleinman, S. J., \& Nitta, A. 2009a, J. Phys. Conf. Ser., 172, 012006

Koester, D., Voss, B., Napiwotzki, R., Christlieb, N., Homeier, D., Lisker, T., Reimers, D., \& Heber, U. 2009b, A\&A, 505, 441

Lang, K. R. 1980, Astrophysical Formulae. A Compendium for the Physicist and Astrophysicist (Berlin: Springer)

Livio, M. 1983, A\&A, 121, L7

Livio, M., \& Pringle, J. E. 1998, ApJ, 505, 339

Lyne, A. G., et al. 1998, MNRAS, 295, 743

Marsh, T. R., Gaensicke, B. T., Steeghs, D., Southworth, J., Koester, D., Harris, V., \& Merry, L. 2010, arXiv:1002.4677

Maxted, P. F. L., \& Marsh, T. R. 1999, MNRAS, 307, 122

Mullally, F., Badenes, C., Thompson, S. E., \& Lupton, R. 2009, ApJ, 707, L51

Nauenberg, M. 1972, ApJ, 175, 417

Nelemans, G., et al. 2005, A\&A, 440, 1087

Oke, J. B. 1990, AJ, 99, 1621

Oke, J. B., et al. 1995, PASP, 107, 375

Patterson, J. 1994, PASP, 106, 209

Phinney, E. S. 1991, ApJ, 380, L17

Pols, O. R., Schroder, K., Hurley, J. R., Tout, C. A., \& Eggleton, P. P. 1998, MNRAS, 298, 525

Pylyser, E., \& Savonije, G. J. 1988, A\&A, 191, 57

Schmidt, G. D., Stockman, H. S., \& Smith, P. S. 1992, ApJ, 398, L57

Sion, E. M., Holberg, J. B., Oswalt, T. D., McCook, G. P., \& Wasatonic, R. 2009, AJ, 138, 1681

Thompson, T. A., Kistler, M. D., \& Stanek, K. Z. 2009, arXiv:0912.0009

Townsley, D. M., \& Bildsten, L. 2004, ApJ, 600, 390

Tremblay, P., \& Bergeron, P. 2009, ApJ, 696, 1755

Tremblay, P., Bergeron, P., Kalirai, J. S., \& Gianninas, A. 2010, ApJ, 715, 172

Tug, H. 1977, The Messenger, 11, 7

van Kerkwijk, M. H., Bassa, C. G., Jacoby, B. A., \& Jonker, P. G. 2005, in ASP Conf. Ser. 328, Binary Radio Pulsars, ed. F. A. Rasio \& I. H. Stairs (San Francisco, CA: ASP), 357

van Kerkwijk, M. H., \& Kaplan, D. L. 2007, Ap\&SS, 308, 191

Vennes, S., Kawka, A., Vaccaro, T. R., \& Silvestri, N. M. 2009, A\&A, 507, 1613

Webbink, R. F., Rappaport, S., \& Savonije, G. J. 1983, ApJ, 270, 678

Wood, M. A. 1992, ApJ, 386, 539

York, D. G., et al. 2000, AJ, 120, 1579 